\title{
PROBLEMÁTICA CONSTITUCIONAL EN LAS ACTIVIDADES EDUCATIVAS Y DEPORTIVAS EN EL MEDIO NATURAL*
}

\author{
ANTONIO BAENA-EXTREMERA*
}

\section{Deporte y Medio ambiente en España}

Luque, Baena-Extremera y Granero-Gallegos ${ }^{1}$, afirman que se está produciendo un importante cambio en la sociedad desarrollada en la que vivimos y en la que se celebran continuamente distintos eventos deportivos, muchos de ellos en el medio natural. En esta sociedad desarrollada, comienzan a converger dos elementos esenciales, con regulaciones legislativas y constitucionales muy diferentes, como son el deporte y el medio ambiente.

España es un país con un desarrollo deportivo importante, y este desarrollo, a veces, no va unido al respeto del medio en el que se lleva a cabo. A nivel ambiental, España cuenta con una gran cantidad de zonas medioambientales protegidas y de interés biológico, aunque, sin duda, no del nivel que cuentan otros países como Chile o Costa Rica. Por ello, en este pequeño país de Europa se comienza a dar desde hace ya alguna década una gran trifurca constitucional entre los límites de ambos conceptos, en pro de buscar la correcta conexión y respeto entre ellos.

Cuando hablo de sociedad desarrollada, moderna, me refiero, siguiendo a los autores citados, a una sociedad preocupada por el entorno, por lo ecológico, por lo rural, por el respeto al medio ambiente,... en definitiva, a una sociedad que debería estar basada en el "Desarrollo Sostenible", pues actualmente, incluso a nivel legal, es lo que se persigue desde los niveles más básicos de

\footnotetext{
* Trabajo recibido el 25 de enero y aprobado el 25 de junio de 2013.

** Licenciado en Educación Física por la Universidad de Granada (España); Doctor en Educación Física por la Universidad de Granada (España); Profesor de la Universidad de Murcia (España). Dirección: C/ Argentina s/n, Santiago de la Ribera (Murcia, España). Teléfono: 0034-619342694. Correo electrónico: abaenaextrem@um.es. El autor agradece enormemente la colaboración de diversos magistrados españoles especialistas en derecho constitucional y otro especialista en medio ambiente que han aportado y colaborado en la elaboración de este trabajo, y que por voluntad propia, prefieren mantenerse en el anonimato.

${ }^{1}$ Luque, Pablo; Baena-Extremera, Antonio y Granero-Gallegos, Antonio, "Buenas prácticas para un desarrollo sostenible en los eventos deportivos en el medio natural", Interciencia: Revista de ciencia y tecnología de América, 36-7, 2011, pp. 531-537.
} 
nuestra educación. Pero, ¿a qué nos referimos con Desarrollo Sostenible? y ¿qué supone a nivel constitucional?

De acuerdo al Informe Brundtland de la Comisión Mundial sobre medio ambiente y desarrollo de las Naciones Unidas, se entiende como "el desarrollo que satisface las necesidades del presente, sin comprometer la capacidad de las generaciones futuras para satisfacer sus propias necesidades ${ }^{\prime 2}$. Gomes ${ }^{3}$ afirma que la sostenibilidad podría ir enfocada en varias dimensiones, como la social, ecológica, económica, espacial, política y cultural. Tanta importancia ha adquirido esta situación que según Lozano ${ }^{4}$ la sostenibilidad ha llegado a atribuirse como un nuevo principio de derecho internacional.

Desde la perspectiva del ámbito deportivo, se entiende la sostenibilidad tal como se indica en el art. 10 de la Carta Europea del Deporte ${ }^{5}$, que dice:

"Garantizar y mejorar el bienestar físico, social y mental de la gente de una generación a la siguiente requiere que las actividades deportivas, incluyendo zonas urbanas, rurales y marítimas, se adapten a los recursos limitados del planeta y se lleven a cabo conforme a los principios de desarrollo sostenido y administración del medio ambiente. Éstos incluyen:

- Tener en cuenta los valores de la naturaleza y del medio ambiente en la planificación y construcción de instalaciones deportivas.

- Apoyo y estímulo a las organizaciones deportivas en sus esfuerzos por conservar la naturaleza y el medio ambiente.

- Aumento de los conocimientos y de la concienciación de la gente sobre las relaciones entre el deporte y el desarrollo sostenido y su comprensión de la naturaleza".

La importancia de esta unión se debe a que la práctica física y deportiva en el medio natural puede conllevar ventajas e inconvenientes ${ }^{6}$. El citado autor destaca entre las primeras ventajas: el incremento del valor recreativo de la zona; el servir como elemento de integración social, o la generación de una conciencia de protección de la naturaleza entre los practicantes. Entre las desventajas, el autor observa cómo los grandes proyectos de infraestructuras pueden causar daños estéticos y ecológicos; también pueden afectar a la fauna y la flora negativamente, o la generación de residuos.

\footnotetext{
${ }^{2}$ Fullana, Pere y Ayuso, Silvia, "Las actividades deportivas en la naturaleza. Evaluación de sus impactos ambientales para alcanzar su sostenibilidad", Curso de Hostelería y Medio Ambiente. Dirección General de Desarrollo Sostenible, Xunta de Galicia. Sanxenxo (Pontevedra), 2004, p. 27.

${ }^{3}$ Gomes, Adriana, "Competitividade e sustentabilidade: é possivel as cidades turisticas sererm sustentáveis e competitivas ao mesmo tempo?", Pasos. Revista de Turismo y Patrimonio Cultural, 4-3, 2006, pp. 443-446.

${ }^{4}$ LozAno, Blanca, Derecho ambiental administrativo, Editorial Dykinson, Madrid, 2007.

${ }^{5}$ Carta Europea del Deporte, de 15 de mayo de 1992.

${ }^{6}$ Ortego, María del Carmen, "Medio Ambiente de Montaña", en Certificado de Iniciación al Montañismo. Texto Oficial del Primer Nivel de Enseñanza de la Escuela Española de Alta Montaña, VV.AA., Huesca, Cuarte, Barrabes Editorial, 2001, pp. 21-52.
} 
Por último, es necesario mencionar la Carta Española de las Montañas, elaborada en 2002 con motivo del año internacional de las montañas, en la cual se recogió la necesidad de que las actividades de ocio y deportivas en los espacios de montaña estuvieran integradas en estrategias más amplias de desarrollo local de los pueblos y sus espacios, cumpliendo, obviamente, con todos los requisitos legales.

Pues bien, una vez entendida esta problemática, vamos a tratar los aspectos constitucionales relacionados con esta conexión.

\section{Deporte, medio ambiente y Constitución española. Planteamiento Del PROBLEMA}

El medio ambiente está recogido en la Constitución española como un principio rector de la política social y económica. Estos principios rectores son conocidos en España como "derechos sociales", y su naturaleza jurídica es diferente del resto de derechos y libertades regulados en la Constitución Española. De esta forma, estos derechos sociales no son derechos subjetivos, por lo que no pueden invocarse ante un juez o tribunal por sí mismos, sino sólo en términos de las posibles leyes que los desarrollen. De ahí que en el art. 53.3 se especifique que "el reconocimiento, el respeto y la protección de los principios reconocidos en el capítulo tercero, informará la legislación positiva, la práctica judicial y la actuación de los poder es públicos. Sólo podrán ser alegados ante la jurisdicción ordinaria de acuerdo con lo que dispongan las leyes que los desarrollen".

Dentro de estos principios rectores, en el art. 45 de la Constitución Española de 1978 se hace patente la necesidad de cuidar la calidad de este medio al recoger en él que:

"1. Todos tienen derecho a disfrutar de un medio ambiente adecuado para el desarrollo de la persona, así como el deber de conservarlo.

2. Los poderes públicos velarán por la utilización racional de todos los recursos naturales, con el fin de proteger y mejorar la calidad de la vida y defender y restaurar el medio ambiente, apoyándose en la indispensable solidaridad colectiva.

3. Para quienes violen lo dispuesto en el apartado anterior, en los términos que la ley fije se establecerán sanciones penales o, en su caso, administrativas, así como la obligación de reparar el daño causado".

Como bien es sabido, el derecho que todo ciudadano tiene de disfrutar el medio ambiente se encuentra ubicado en la Constitución dentro de los Principios Rectores de la Política Social y Económica del Capítulo III del Título $1^{\circ}$ que, según establece el art. 53.3., "informarán la legislación positiva, la práctica judicial y la actuación de los poderes públicos. Sólo podrán ser alegados ante la jurisdicción ordinaria de acuerdo con lo que dispongan las leyes que 
lo desarrollen". Sin embargo, como ya afirma Calderón ${ }^{7}$, estos derechos no son susceptibles de amparo constitucional.

En España, el Tribunal Constitucional publicó una sentencia ${ }^{8}$, donde se hace mención a la configuración específica constitucional de este derecho, especificando claramente que "el derecho al medio ambiente adecuado revista una singular importancia, acrecentada en la sociedad industrializada y urbanizada de nuestros días (...) sin embargo no puede ignorarse que el art. 45 de la Constitución enuncia un principio rector, no un derecho fundamental. Los tribunales deben velar por el respeto al medio ambiente, sin duda, pero de acuerdo con lo que dispongan las leyes que desarrollan el precepto constitucional". Siguiendo esta idea, el Tribunal Constitucional español publicó otra sentencia ${ }^{9}$ en la que se resolvieron diversos recursos de inconstitucionalidad y de conflictos de competencia acumulados en relación con la ley ${ }^{10}$ de conservación de los espacios naturales y el de flora y fauna silvestre.

En esta línea, así como según las manifestaciones de varios autores como Lagardera ${ }^{11}$, no se trataría de eliminar el deporte en el medio ambiente, sino de un progreso en el proceso de civilización y en el proceso de educación; es lo que Maffesoli12 denomina una "ecologización" del mundo, y dentro de nuestro mundo, el deporte es uno de ellos; "la naturaleza ya no es sólo un objeto a explotar, sino que se convierte en un compañero imprescindible".

En España existe una tendencia ecológica importante debida, entre otras consideraciones, a los espacios naturales que dispone este país, y también, por el aumento de la población que acude a estas zonas en su tiempo de ocio y descanso. Así, se puede entender que la doctrina de ecologización de los derechos fundamentales reconocidos en los arts. 15 y 18, y más concretamente ante la posibilidad de producir ciertas agresiones al entorno, debe ser tenida en cuenta como principio de las posibilidades de agredir a la vida humana si ésta se ve a afectada dentro del equilibrio natural.

\footnotetext{
7 CAlDerón, José María, "Tributación medioambiental en las comunidades autónomas y entidades locales. Especial referencia a las emisiones atmosféricas", en Consejo General del Poder Judicial (edit.), Instrumentos judiciales de fomento para la protección del medioambiente, Madrid, Lepko Print, 2005, pp. 61-163.

${ }^{8}$ STC No 199/1996 de 3 de diciembre.

${ }^{9}$ STC No 102/1995 de 26 de junio.

${ }^{10}$ Ley 4/1989 de 27 de marzo, de Conservación de los Espacios Naturales y el de Flora y Fauna Silvestre.

${ }^{11}$ Lagardera, Francisco, "Desarrollo sostenible en el deporte, el turismo y la educación física", Apunts, Educación Física y Deportes, 67, 2002, pp. 70-79.

12 Maffesol, Michel, "La socialidad en la posmodernidad", en Vattimo, Gerardo et al., En torno a la posmodernidad, Barcelona, Anthropos, 1990, pp. 193-110.
} 
Al respecto, siguiendo la doctrina del caso López Ostra ${ }^{13}$, el citado tribunal hizo mención reiterada a la misma el 19 de febrero de 1998 en el caso Guerra contra Italia, a raíz de emisiones tóxicas provenientes de una fábrica cercana; resolución en que el alto tribunal señala "que los atentados graves en el ambiente pueden afectar el bienestar de las personas y privarles de disfrute...".

De la propia definición del art. 45 de la Constitución se deduce que el medio ambiente afecta a todos los recursos naturales necesarios para preservar la calidad de vida de los seres humanos, como las aguas, el aire, la flora... No obstante, el Tribunal Constitucional ha declarado que éste se integra, además de los recursos naturales, por otros elementos importantes, que presentan una naturaleza de carácter histórico, monumental, como es el "paisaje", constituyendo este último una "noción de estética", cuyos ingredientes son naturales -tierra, valle, mar, etc-.

Desde esta perspectiva, el valor del medio ambiente presenta otros problemas a la hora de su coordinación con la defensa de otros derechos que en la Constitución Española también se reconocen, como es el reconocimiento de la libertad de empresa. La libertad de empresa se enmarca dentro del capítulo II de la Constitución Española de "derechos y libertades", auténtica declaración de derechos, capítulo que, a su vez, se divide en dos secciones; una primera relativa a los derechos fundamentales y de libertades públicas, y una segunda donde se recogen los derechos y deberes de los ciudadanos, ambos presididos por el principio de igualdad (art. 14). En dicha sección segunda se incluye la libertad de empresa como un derecho constitucional, indicando en el art. 38 que: "se reconoce la libertad de empresa en el marco de la economía de mercado. Los poderes públicos garantizan y protegen su ejercicio y la defensa de la productividad, de acuerdo con las exigencias de la economía general y en su caso, de la planificación".

Pues bien, a partir de aquí el problema que se plantea es cómo poder hacer uso legítimo de ambos, de manera que la libertad de empresa no conlleve en ocasiones la creación de infraestructuras que perjudiquen y deterioren el medio ambiente. Por tanto, esta tensión se produce en las ocasiones en las que la creación de una nueva construcción dentro de un entorno ambiental para practicar deportes y actividades en la naturaleza, supone el deterioro e impacto de alguna zona de especial valor medioambiental. En España, y cada vez con mayor frecuencia, la proliferación de empresas que se dedican a ofertar actividades en la naturaleza se adueñan a través de convenidos y contratos con ayuntamiento, de zonas de ambientales que luego no conservan ni respetan como las leyes de

${ }^{13}$ STEDH No 496/1994 de 9 de diciembre. 
lugar establecen. Por ese motivo Camps, Carretero y Perich ${ }^{14}$ afirman que esta reglamentación afecta en unos casos a las estructuras, en otros a las actividades $y$, en tercer lugar, puede afectar a las personas que viven en esos lugares, por el deterioro del medio que les rodea. Ante esta situación no existe una técnica jurídica de resolución única, pues, ante todo, habría que conocer el alcance de la empresa, el ordenamiento y protección del territorio, las actividades a llevar a cabo, la legislación autonómica y estatal de protección medioambiental, etc. Todo esto supone hoy en día un vacío y un problema que está en auge.

\section{JURISPRUDENCIA DE LA MATERIA}

A nivel europeo, la protección del medio ambiente no se recoge de forma explícita por el Tribunal Europeo de Derechos Humanos creado en 1950, ya que en aquella fecha no existía la necesidad de elevar el medio ambiente a la categoría de un derecho humano. Así, en el título I del Convenio europeo para la protección de los derechos humanos y libertades fundamentales o Convenio europeo de derechos humanos, se establecen aquellos que son susceptibles de protección, entre los que no se encuentra el derecho al medio ambiente. Esta deficiencia resulta compensada por el establecimiento de un mecanismo de protección consistente en un sistema judicial internacional al cual el individuo tiene acceso directo bajo determinadas condiciones. Por lo tanto, a pesar de que el derecho humano al medio ambiente no está recogido de modo expreso en el sistema del Convenio, este derecho podría ser protegido por el Tribunal si dicho órgano considerase que se encuentra garantizado de forma implícita en algunos de los derechos enunciados en el sistema del Convenio. Como consecuencia de la evolución de esta vía de protección indirecta, surge una de las grandes líneas jurisprudenciales del Tribunal Europeo de Derechos Humanos: la protección del derecho a un medio ambiente adecuado. No obstante, ante el caso que estamos discutiendo, no existe una tendencia unánime de actuación, ni ninguna sentencia ejemplar que clarifique este caso.

Por tanto, el análisis del derecho constitucional o del ciudadano al medio ambiente a nivel europeo no se da de manera directa, sino a través de la protección de los derechos que se enumeran en el Convenio. Por este motivo, a nivel jurisprudencial escasean los casos donde se produzca la problemática que en este trabajo se aborda. No obstante, siguiendo la línea anterior, el Tribunal Europeo de Derechos Humanos ${ }^{15}$ establece como máxima de interpretación

\footnotetext{
${ }^{14}$ Camps, Andreu; Carretero, José Luis y Perich, María Jesús, "Aspectos normativos que inciden en las actividades físico-deportivas en la naturaleza", Apunts: Educación Física y Deportes, 45, 1995, pp. 44-52.
}

${ }^{15}$ STEDH No $16281 / 2010$ de 30 de marzo. 
y protección el derecho a la vida y al domicilio (fundamentales) respecto a las actividades peligrosas en conexión con su/el impacto medioambiental que implica para el derecho fundamental (incidencia de actividades peligrosas en el medio ambiente para la protección de la vida). Esta sentencia recordó que los atentados graves contra el medio ambiente pueden afectar al bienestar de las personas y privarlas del disfrute de su domicilio y de su vida. Por ese motivo, los estados tienen la obligación de controlar la autorización, funcionamiento, explotación seguridad y control de las actividades peligrosas y de asegurar la protección efectiva de los ciudadanos en los casos en los que la vida se puede poner en peligro como consecuencia de tales actividades.

En España, y teniendo en cuenta la situación planteada, el Tribunal Constitucional se ha referido a estos artículos, en diversas ocasiones, y a su enfrentamiento expresando que "existe una necesidad de compaginar en la forma que en cada caso decida el legislador competente la protección de ambos bienes constitucionales: el medio ambiente y el desarrollo económico", tal como queda reflejado en Sentencia ${ }^{16}$. Como entendemos, el desarrollo económico de dichas zonas se puede ver favorecido por la creación de empresas, o no.

En relación a la jurisprudencia existente, es interesante destacar algunas sentencias que dejan patente esta problemática. Por ejemplo, la STSJ de Castilla y León ${ }^{17}$, estimó el recurso contencioso-administrativo interpuesto por varias asociaciones ecologistas contra el Decreto $N^{0} 56 / 2008^{18}$. Este parque de ocio se aprobó previamente sin tener la vista favorable sobre el impacto ambiental que iba a ocasionar en la zona, y tras modificar su proyecto inicial, éste volvió a presentarse sin dicha aprobación.

Otro ejemplo claro es la STSJ de Galicia ${ }^{19}$, que denegó la autorización de construcción de un hotel de naturaleza en suelo rústico. Dicha sentencia anuló la resolución impugnada dado el régimen transitorio del Decreto No 267/199920, por cuya virtud se permite la construcción en suelo rústico de instalaciones hoteleras respetando las condiciones paisajísticas de la zona y su ubicación destinadas al turismo rural. Dicha sentencia explica claramente que la construcción, en este caso, de un negocio donde se van a practicar a su alrededor actividades en la naturaleza debe respetar el entorno donde se ubica y, concretamente, la ley de protección medioambiental de dicha zona. Por tanto, la libre

\footnotetext{
${ }^{16}$ STC 64/1982 de 4 de noviembre.

17 STS Castilla y León № 5319/2012, de 13 de julio.

${ }^{18}$ Decreto No 56/2008, de 31 de julio, de la Junta de Castilla y León, por el que se aprueba el Proyecto Regional para la ejecución de un Parque de Ocio en el municipio de Arlanzón (Burgos).

${ }^{19}$ STSJ Galicia No 1236/2010 de 11 marzo.

${ }^{20}$ Decreto № 267/1999, de 30 de septiembre, por el que se establece la ordenación de los establecimientos hoteleros.
} 
empresa no puede perjudicar dicho medio, sino, todo lo contario, protegerlo y conservarlo.

Siguiendo el caso anterior, la STSJ Cataluña ${ }^{21}$, multó a la empresa Motor Club Terrassa por la celebración de una prueba dentro del campeonato de Cataluña de Enduro por un itinerario distinto al autorizado sin que, además, se hubieran reparado, después de transcurridos 30 días, los daños causados. La causa principal de este acto fue la controversia relativa a la libre práctica de actividades empresariales en lugares protegidos sin que exista un procedimiento previo que apruebe los mecanismos de reducción del impacto ambiental de la zona.

En ese mismo año, otro ejemplo que se puede considerar como una sentencia pionera es la STSJ Castilla y León ${ }^{22}$. En ella se estima la demanda interpuesta por la asociación para la defensa de los recursos naturales de Cantabria contra la modificación de una norma que permitiría la construcción de estaciones de esquí alpino por un grupo de empresarios sin que la Administración Autonómica se haya asegurado de que no causará perjuicio a la integridad del parque natural, debido a su fragilidad ambiental. A grandes rasgos, dicha sentencia fue la primera en utilizar el cambio climático en sus argumentaciones y anuló la modificación del Plan de Ordenación de los Recursos Naturales (PORN) de un parque natural, con la que se pretendía abrir la posibilidad de autorizar en el mismo, la explotación por una empresa a través de la creación de estaciones de esquí alpino. Pero no es el único ejemplo, pues, además de la nombrada, existen otras dos sentencias del TSJ de Castilla y León ${ }^{23}$ que anulan el Decreto $N^{0} 13 / 2006^{24}$, con el fin de dar cabida a la estación de esquí.

Todos estos ejemplos dan muestra de la problemática existente entre la libre empresa y el respeto al medio natural. No obstante, existen muestras claras de entendimiento entre ambos artículos. Un ejemplo bastante reciente es la Resolución 13 de 13 de noviembre de $2012^{25}$, a partir de la cual se resuelve no someter al procedimiento de evaluación de impacto ambiental el proyecto de una empresa de aventura. Esto se debe fundamentalmente a que muchas de las empresas optan por buscar dos soluciones: ubicarse en zonas naturales con

\footnotetext{
${ }^{21}$ STS Cataluña No 251/2008 de 28 marzo.

22 STSJ Castilla y León No 20/2008 de 8 enero.

${ }^{23}$ STS Castilla y León № 1158/2008 de 27 mayo y STS Castilla y León № 1636/2008 de 4 julio.

${ }^{24}$ Decreto № 13/2006 que hizo la Junta para modificar el Plan de Ordenación de los Recursos Naturales (PORN) del Parque Natural de Fuentes Carrionas.

${ }^{25}$ Resolución № 13 de noviembre de 2012, del Instituto Aragonés de Gestión Ambiental, por la que se resuelve no someter al procedimiento de evaluación de impacto ambiental el proyecto de instalación de un parque aventura en el municipio de Torres de Albarracín (Teruel), promovido por Deporte Aventura Sierra de Albarracín, S.L.
} 
bajo interés natural, y proponer actividades deportivas y de aventura de bajo impacto ambiental.

Lo que está claro en este asunto es que la relación entre la libre empresa y el respeto al medio natural debe darse en un marco de coherencia entre ambos, pues parte del desarrollo económico de dichas zonas se ve acrecentado por estos negocios. Esto nos hace entender que el desarrollo de una zona con importancia medioambiental es un proceso localizado de cambio social y crecimiento económico sostenible que tiene por finalidad el progreso permanente de la comunidad rural y de cada individuo integrado en ella. Así, siguiendo a Gómez ${ }^{26}$, puede entenderse en un sentido básico como la mejora de las condiciones de vida de los habitantes de los espacios rurales, fijando objetivos como la mejora de la calidad de vida de los mismos y el incremento, a su vez, de los niveles económicos, las condiciones de vida y de trabajo y la conservación del medio ambiente.

Como ya se sabe, el desarrollo económico de una zona y su progreso industrial, cultural y social está unido insoslayablemente al de su economía, y no cabe duda de que llevar a cabo un desarrollo sostenido estaría basado en la utilización racional, empleando los términos del art. 45 de la Constitución Española, de los recursos naturales y, por tanto, en la protección del medio ambiente.

Sobre esto, en una Sentencia ${ }^{27}$, se expresa literalmente: "para ello, hemos de remontarnos a la calidad de vida como aspiración situada en primer plano por el Preámbulo de la Constitución, que en un principio parece sustentarse sobre la cultura y la economía, aun cuando en el texto articulado se ligue por delante a la utilización racional de los recursos naturales y por detrás al medio ambiente con el trasfondo de la solidaridad colectiva. En suma, se configura un derecho de todos a disfrutarlo y un deber de conservación que pesa sobre todos, más un mandato a los poderes públicos para la protección (art. 45 CE). En seguida, la conexión indicada se hace explícita cuando se recomienda a los Poderes públicos para la función de impulsar y desarrollar se dice, la actividad económica y mejorar así el nivel de vida, ingrediente de la calidad si no sinónimo, con una referencia directa a ciertos recursos (la agricultura, la ganadería, la pesca) y a algunos espacios naturales (zonas de montaña) (art. 130 (E), lo que nos ha llevado a resaltar la necesidad de compatibilizar y armonizar ambos, el desarrollo con el medio ambiente ${ }^{28}$. Se trata en definitiva del 'desarrollo sostenible', equilibrado y racional, que no olvida a las generaciones futuras, alumbrando el año 1987 en el Ilamado Informe Bruntland,

\footnotetext{
${ }^{26}$ GOMEs, "Competitividade", cit. nota n. 3.

${ }^{27}$ STC No 102/1995 de 26 de junio.

${ }^{28}$ STC 64/1982 de 4 de noviembre.
} 
con el título "Nuestro futuro común", encargado por la Asamblea General de las Naciones Unidas".

Así pues, la voluntad de utilizar el ordenamiento jurídico en general, y el tributario en particular, en aras de contribuir a la protección del medio ambiente se halla, por otra parte, en perfecta sintonía con las más modernas materializaciones del marco comunitario ${ }^{29}$.

De esta manera, es dentro de este ámbito donde es determinante la actuación de los medios para permitir llevar a cabo una evaluación de los costes medioambientales de la actividad económica efectuada, incentivando aquéllas que sean sostenibles, a la vez que repercutiendo los gastos de degradación del medio en los capítulos de costes de producción de las empresas, con arreglo al principio de quien contamina paga, todo ello, basado en el principio de "la indispensable solidaridad colectiva", que deja expreso el art. 45 de la Constitución Española.

Este principio de que "quien contamina paga" está incorporado por el Acta Única Europea al Tratado de la Comunidad Europea, y constituye un principio esencial de la acción comunitaria y significa, simplemente, que los costes de la contaminación se han de imputar al que provoca la misma. Pero, en cambio, en ocasiones las empresas no producen contaminación, pero si deterioro del medio, y esto ¿cómo se paga? Por todo ello, creo que los poderes legislativos deberían tener además muy en cuenta el principio de "acción preventiva y de cautela", que significa que las medidas de protección del medio ambiente se han de adoptar aún antes de que se haya producido una lesión al medio ambiente.

Desde la perspectiva de la distribución competencial, según la Constitución corresponde a las comunidades autónomas la promoción y ordenación del turismo en sus ámbitos territoriales (art. 148.1.18 a y la promoción del deporte y de la adecuada utilización del ocio (art. 148.1.19ª). Así, se recoge en el art. 149.1.23 que se atribuye al estado la competencia exclusiva sobre "la legislación básica sobre protección del medio ambiente, sin perjuicio de las facultades de las comunidades autónomas de establecer normas adicionales de protección", y en el art. 148.1.9, permite que sean las comunidades autónomas las que asuman competencias en "la gestión en materia de protección del medio ambiente". En esta línea, y en relación a la protección del medio ambiente, es interesante tener en cuenta la $\mathrm{STC}^{30}$, donde se puede observar como se estimó el recurso de inconstitucionalidad interpuesto contra la Ley del Parlamento Vasco ${ }^{31}$. Igual-

\footnotetext{
${ }^{29}$ Calderón, "Tributación", cit. nota n. 7.

${ }^{30}$ STC No 101/2006 de 30 de marzo.

${ }^{31}$ Ley del Parlamento Vasco № 3/1998, de 27 de febrero, General de Protección del Medio Ambiente del País Vasco.
} 
mente, esta situación de conflicto entre estado y autonomía se da en la STC ${ }^{32}$ sobre el parque natural de Doñana (Andalucía), en la STC de $20 \mathrm{abril}^{33}$ o en la STC de 17 febrero ${ }^{34}$, ambas del 2005.

Por tanto, la solución propuesta para este dilema es doble:

- Por un lado, que el poder judicial establezca formas claras para hacer fácil la relación entre ambos artículos, sin perjuicio de ninguno de ellos y en pro de una conservación sostenible del medio y de la práctica deportiva a través de empresas.

- Por otro lado, que las comunidades autónomas establezcan tributos extrafiscales específicamente orientados a la protección del medio ambiente, así como que se fijen incentivos ecológicos en aquellos tributos autonómicos de carácter ordinario. Por lo tanto, a la naturaleza habría que ponerle un precio, algo que actualmente se hace, pero que es difícil de estimar con coherencia, ¿o no?

- Desde mi punto de vista es una cuestión compleja de resolver, pues sería imprescindible conocer la misión de dicha empresa y la normativa autonómica, nacional y europea de protección medioambiental que pueda afectar en cada caso. Por todo esto, la posible solución dependería de dichas variables, y de la interpretación jurídica de cada magistrado.

\section{COnClusiones}

Como bien expresa Nogueira ${ }^{35}$, existe una conciencia sobre la necesidad de proteger el medio o entorno, buscando el modo de sobrevivir en este planeta. Para ello, hay que buscar un equilibrio entre los recursos naturales renovables y no renovables que posibiliten el mantenimiento de un ambiente propicio a la mantención del desarrollo humano y una cierta calidad de vida humana. Y el deporte, no debe ser el elemento que perturbe este equilibrio, y menos aún, que conlleve una sobre explotación y deterioro del medio ambiente.

La sociedad postmoderna está cambiando, y uno de sus reflejos es el aumento de la actividad físico-deportiva en el medio natural. Consecuencia de ello es el aumento de los posibles impactos ambientales. La administración central y autonómica y la legislación estatal se va haciendo poco a poco "eco" de las actividades físicas en el medio natural y de sus posibles impactos ambientales. Cada vez son más los estamentos educativos y deportivos que recogen en sus normas el respeto al medio ambiente como un principio básico para la orga-

\footnotetext{
${ }^{32}$ STC No 331/2005 de 15 diciembre.

${ }^{33}$ STC No 101/2005 de 20 abril.

${ }^{34}$ STC No 33/2005 de 17 febrero.

${ }^{35}$ Nogueira, Humberto, Revista Jurídica Justicia ambiental. Revista de derecho ambiental de la fiscalía del medio ambiente (FIMA), Estudios Constitucionales, 7-2, 2009, pp. 467-474.
} 
nización y/o participación en las actividades físicas en el medio natural y en sus respectivos eventos deportivos. Pero aún falta mucho, tal como expresan Luque et al. ${ }^{36}$

Esta problemática exige planteamientos legales acordes con la gravedad de la misma, además de una conciencia de este asunto por parte de los practicantes, organizadores, espectadores y, por supuesto, legisladores. El reflejo de esta toma de conciencia está en la ambientalización de las prácticas deportivas en general y de los de naturaleza en particular. Un ejemplo claro lo podemos ver en el Decreto de la Generalitat de Cataluña en España ${ }^{37}$, donde se obliga a las empresas que trabajan en el medio ambiente a establecer unas medidas para evitar el impacto ambiental y otras para incidir en sus clientes informándoles debidamente antes de cada actividad.

A modo de conclusión, quisiera finalizar este trabajo planteando una reflexión individual, dada la importancia de la cuestión que nos ocupa. Debemos preguntarnos si la libertad de empresa recogida en la Constitución española conlleva un aumento en los acercamientos de la población al medio ambiente realizándose en simbiosis con ella. $\mathrm{O}$, por el contrario, trasladamos a la misma, mediante las actividades que realizamos, el modo de vida diario y urbano, sin tener en cuenta dónde pisamos, destruyendo, contaminando y "limpiando" cualquier rastro de vida (fauna y flora) si así es necesario para ciertas prácticas en las que, en muchas ocasiones, el egocentrismo justifica nuestros comportamientos y actitudes. En este caso, el poder judicial en cualquier país donde ocurriera debería tomar parte en este asunto y cuidar del medio en el que vivimos, que es parte de nosotros.

\footnotetext{
${ }^{36}$ Luque, Baena-Extremera y Granero-Gallegos, "Buenas prácticas", cit. nota n. 1.

${ }^{37}$ Decreto No 81/1991 de 25 de marzo de la Generalitat de Cataluña en España.
} 